\title{
European position paper on the management of patients with patent foramen ovale. General approach and left circulation thromboembolism
}

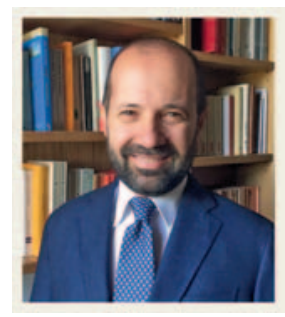

Christian Pristipino ${ }^{1 *}$, Horst Sievert ${ }^{2,3}$, Fabrizio D'Ascenzo ${ }^{4}$, Jean Louis Mas ${ }^{5}$, Bernhard Meier ${ }^{6}$, Paolo Scacciatella ${ }^{4}$, David Hildick-Smith ${ }^{7}$, Fiorenzo Gaita ${ }^{4}$, Danilo Toni ${ }^{8}$, Paul Kyrle ${ }^{9}$, John Thomson ${ }^{10}$, Genevieve Derumeaux ${ }^{11}$, Eustaquio Onorato $^{12}$, Dirk Sibbing ${ }^{13}$, Peter Germonpré ${ }^{14}$, Sergio Berti ${ }^{15}$, Massimo Chessa ${ }^{16}$, Francesco Bedogni ${ }^{16}$, Dariusz Dudek ${ }^{17}$, Marius Hornung ${ }^{2}$, and Jose Zamorano ${ }^{18}$, joint task force of European Association of Percutaneous Cardiovascular Interventions (EAPCI), European Stroke Organisation (ESO), European Heart Rhythm Association (EHRA), European Association for Cardiovascular Imaging (EACVI), Association for European Paediatric and Congenital Cardiology (AEPC), ESC Working group on GUCH, ESC Working group on Thrombosis, European Haematological Society (EHA), European Underwater and Baromedical Society (EUBS)

Evidence Synthesis Team: Fabrizio D'Ascenzo ${ }^{4}$, Pierluigi Omedè ${ }^{4}$, Flavia Ballocca ${ }^{4}$, Umberto Barbero ${ }^{4}$, Francesca Giordana ${ }^{4}$, Sebastiano Gili ${ }^{4}$, and Mario lannaccone 4

Eapci Scientific Documents and Initiatives Committee:

Davide Capodannno ${ }^{19}$, Marco Valgimigli ${ }^{6}$, and Robert Byrne ${ }^{20}$

International Experts: Teiji Akagi ${ }^{21}$, John Carroll ${ }^{22}$, Bharat Dalvi ${ }^{23}$, Junbo $\mathrm{Ge}^{24}$, Scott Kasner ${ }^{25}$, Ina Michel-Behnke ${ }^{26}$, Carlos Pedra ${ }^{27}$, John Rhodes ${ }^{28}$, Lars Søndergaard $^{29}$, Lars Thomassen ${ }^{30}$, and Giuseppe G.L. Biondi-Zoccai ${ }^{31,32}$

${ }^{1}$ S. Filippo Neri Hospital - ASL Roma 1, Rome, Italy; ${ }^{2}$ CardioVascular Center (CVC) Frankfurt, Frankfurt, Germany; ${ }^{3}$ Anglia Ruskin University, Chelmsford, United Kingdom, and University of California San Francisco (UCSF), San Francisco, USA; ${ }^{4}$ Città della Salute e della Scienza Hospital, University of Turin, Turin, Italy; ${ }^{5}$ Hôpital Sainte-Anne, Université Paris Descartes, Paris, France; ${ }^{6}$ University Hospital, Bern, Switzerland; ${ }^{7}$ Sussex Cardiac Centre, Brighton and Sussex University Hospitals, Brighton, United Kingdom; ${ }^{8}$ Hospital Policlinico Umberto I, Sapienza University, Rome, Italy; ${ }^{9}$ Medical University, Vienna, Austria; ${ }^{10}$ Leeds General Infirmary, Leeds, United Kingdom; ${ }^{11}$ Faculté de Médecine de Créteil, Hôpital Henri Mondor, Créteil, France; ${ }^{12}$ Humanitas Gavazzeni, Bergamo, Italy; ${ }^{13}$ Campus Großhadern, Ludwig-Maximilians-Universität (LMU), Munich, Germany; ${ }^{14}$ Military Hospital, Brussels, Belgium; ${ }^{15}$ Heart Hospital, Massa, Italy; ${ }^{16}$ Policlinico, San Donato, University Hospital, San Donato Milanese, Milan, Italy; ${ }^{17}$ University Hospital, Krakow, Poland;

* Corresponding author: San Filippo Neri - ASL Roma 1 Hospital, Via Alessandro Poerio 140, 00152 Rome, Italy. E-mail: pristipino.c@gmail.com H. Sievert and F. D'Ascenzo contributed equally to the manuscript

This paper was guest edited by David R. Holmes, MD, MACC,. Department of Cardiology, Mayo Clinic, Rochester, MN, USA.

The article has been co-published with permission in the European Heart Journal and Eurolntervention. All rights reserved. ๑ The Author(s) 2018. The articles are identical except for minor stylistic and spelling differences in keeping with each journal's style. Either citation can be used when citing this article. 


\begin{abstract}
${ }^{18}$ University Hospital Ramón y Cajal, Madrid, Spain; ${ }^{19}$ Azienda Ospedaliero-Universitaria "Policlinico-Vittorio Emanuele", University of Catania, Catania, Italy;
${ }^{20}$ Deutsches Herzzentrum München, Technische Universität München, Munich, Germany; ${ }^{21}$ Okayama University Hospital, Okayama, Japan; ${ }^{22}$ University of Colorado Hospital, Denver, CO, USA; ${ }^{23}$ Glenmark Cardiac Centre, Mumbai, India; ${ }^{24}$ Shanghai Institute of Cardiovascular Disease, Shanghai, China; ${ }^{25}$ University of Pennsylvania, Philadelphia, PA, USA; ${ }^{26}$ Kinderherzzentrum University of Vienna, Vienna, Austria; ${ }^{27}$ Dante Pazzanese Instituto de Cardiologia, Sao Paulo, Brazil; ${ }^{28}$ Nicklaus Children's Hospital, Miami, USA, FL; ${ }^{29}$ Rigshospitalet, Copenhagen, Denmark; ${ }^{30}$ Haukeland University Hospital, Bergen, Norway; ${ }^{31}$ Sapienza University of Rome, Latina, Italy ${ }^{32}$ Department of AngioCardioNeurology, IRCCS Neuromed, Pozzilli, Italy
\end{abstract}

Received 24 September 2018; accepted 28 September 2018

The presence of a patent foramen ovale (PFO) is implicated in the pathogenesis of a number of medical conditions; however, the subject remains controversial and no official statements have been published. This interdisciplinary paper, prepared with involvement of eight European scientific societies, aims to review the available trial evidence and to define the principles needed to guide decision making in patients with PFO. In order to guarantee a strict process, position statements were developed with the use of a modified grading of recommendations assessment, development, and evaluation (GRADE) methodology. A critical qualitative and quantitative evaluation of diagnostic and therapeutic procedures was performed, including assessment of the risk/benefit ratio. The level of evidence and the strength of the position statements of particular management options were weighed and graded according to predefined scales. Despite being based often on limited and non-randomised data, while waiting for more conclusive evidence, it was possible to conclude on a number of position statements regarding a rational general approach to PFO management and to specific considerations regarding left circulation thromboembolism. For some therapeutic aspects, it was possible to express stricter position statements based on randomised trials. This position paper provides the first largely shared, interdisciplinary approach for a rational PFO management based on the best available evidence.

\section{Abbreviations}

$\begin{array}{ll}\text { AF } & \text { Atrial fibrillation } \\ \text { AUC } & \text { Area under the receiver operating curve } \\ \text { c-TCD } & \text { Contrast-enhanced transcranial Doppler } \\ \text { c-TOE } & \text { Contrast transoesophageal echocardiography } \\ \text { c-TTE } & \text { Contrast-enhanced transthoracic echocardiography } \\ \text { DOAC } & \text { Direct oral anticoagulants } \\ \text { DVT } & \text { Deep vein thrombosis } \\ \text { ECG } & \text { Electrocardiogram } \\ \text { GRADE } & \text { Grading of recommendations assessment, development, } \\ & \text { and evaluation } \\ \text { ICM } & \text { Insertable cardiac monitors } \\ \text { LAE } & \text { Left atrium enlargement } \\ \text { LVH } & \text { Left ventricle hypertrophy } \\ \text { NNH } & \text { Number needed to harm } \\ \text { NNT } & \text { Number needed to treat } \\ \text { OAC } & \text { Oral anticoagulants } \\ \text { OR } & \text { Odds ratio } \\ \text { OSAS } & \text { Obstructive sleep apnoea syndrome } \\ \text { PE } & \text { Pulmonary embolism } \\ \text { PICO } & \text { Population-intervention-comparator-outcome } \\ \text { PFO } & \text { Patent foramen ovale } \\ \text { RCT(s) } & \text { Randomised clinical trial(s) } \\ \text { RoPE } & \text { Risk of paradoxical embolism } \\ \text { R-T-L } & \text { Right-to-left } \\ \text { Rx } & \text { Therapy } \\ \text { TIA } & \text { Transient ischaemic attack }\end{array}$

\section{Introduction}

The presence of a patent foramen ovale (PFO) is implicated in the pathogenesis of a number of medical conditions. Recent randomised clinical trials (RCTs) have shown evidence of benefit for device closure as compared with medical therapy in patients with cryptogenic stroke. However, we are rarely able to be categoric about the role of PFO in any given clinical setting, stressing the need for specific clinical and research approaches for complex scenarios. ${ }^{1-5}$ Moreover, most studies on the subject are observational, with an ensuing low certitude of effects and very disparate, often contradictory, clinical choices in different local realms in the absence of official positions. To address these concerns, the European Association of Percutaneous Cardiovascular Interventions (EAPCl) Scientific Documents and Initiatives Committee invited eight European scientific societies and international experts to develop shared and rational position statements on the management of PFO to help clinicians in decision making. To address that request, this paper aims to define interdisciplinary rational principles needed to guide management of patients with PFO by using a strict methodology to prepare position statements with different underlying quality of evidence, based on systematic literature reviews for each of the considered issues and performing quantitative assessments whenever possible.

The present paper reports the approach to patients with PFO and left circulation thromboembolisms, that affect large numbers of patients. ${ }^{6-8}$ A subsequent paper will report on decompression sickness, desaturation syndromes, migraine, and other clinical settings. 


\section{Methods}

In order to guarantee a strict evidence-based process, position statements were developed with the use of a modified grading of recommendations assessment, development, and evaluation (GRADE) methodology (http://gdt.guidelinedevelopment.org/app/handbook/hand book.html), by answering population-intervention-comparator-outcome (PICO) questions and non-PICO questions.

A detailed review of the methodology used can be found in Supplementary Appendix 1, Supplementary Appendix 2, Supplementary Appendix 3 and Supplementary Table 12. Systematic reviews and statistical analysis were performed by a dedicated evidence synthesis team.

\section{Is PFO associated with cryptogenic left circulation thromboembolism?}

The association between PFO and cryptogenic left circulation thromboembolism has mainly been addressed in studies including cryptogenic stroke and is strongly supported by epidemiological data, ${ }^{9-13}$ clinical observational studies ${ }^{14-25}$ (Supplementary Appendix 4) and by RCTs showing that PFO closure reduces stroke recurrence in comparison with medical therapy. ${ }^{26-29}$

However, the evidence has been controversial due to the different role that a PFO can play in different clinical scenarios and to the lack of adequately dimensioned prospective studies. Pathophysiological processes include paradoxical embolism, thrombus forming within the PFO, left atrial dysfunction, and atrial arrhythmias (Supplementary Appendix 4). Research aimed at identifying individual patients' phenotypes is needed to improve clinical management.

\section{Definitions of PFO-related left circulation thromboembolism}

PFO has been associated with left circulation thromboembolism to several organs; ${ }^{30}$ therefore we promote the use of standardised definitions.

Cryptogenic ischaemic left circulation embolisms are defined as any definite ischaemia (symptomatic or asymptomatic) occurring in an arterial bed which lacks a known cause despite investigation. Patients presenting with this clinical picture should be screened for the presence or absence of a PFO. However, when a PFO is thought likely to be implicated in a cryptogenic embolism, the event should be classified as PFO-related instead of cryptogenic. ${ }^{31}$ Current classifications do not yet generally include this aspect. ${ }^{32-35}$

\section{General approach to PFO management}

The management we propose in this paragraph applies to systemic thromboembolism as well as to all PFO-associated syndromes. An overview of the general approach to PFO management is summarised in Table 1.

\section{The main axes of evaluation}

In all clinical scenarios, the two main axes guiding assessment and treatment of PFO should be: 1) the probability that any PFO has a relevant role in the observed clinical picture; 2 ) the likelihood that the observed clinical event will recur. For patients with the highest probability of both, closure of the PFO should be advised. For patients with the lowest probability, medical therapy should be considered. For patients with intermediate probabilities, clinical judgement is required to allow good decision making in liaison with the patient.
Proactive approach: an interdisciplinary collaboration, shared decision making, and open informed consent

Interdisciplinary involvement in decision making regarding PFO management is axiomatic and should include an interventional cardiologist and other specialists dictated by the patient's clinical manifestations. Active involvement of the patient in the decision-making process is mandatory ${ }^{36,37}$ and should be documented in an individualised, open, informed consent. The development of specific decision aids and the use of narrative tools are encouraged. ${ }^{38-43}$

\section{Diagnosing PFO}

The diagnosis of PFO is required only for deciding on a treatment. Several techniques can be used to diagnose PFO. ${ }^{44}$ Their characteristics are summarised in Supplementary Table 1. High-quality comparative studies are still needed to express a conclusive position on the best diagnostic strategies.

Contrast transoesophageal echocardiography (c-TOE) provides unparalleled visualisation of the interatrial septum and other relevant structures and can show the shunt itself. A meta-analysis of the accuracy of c-TOE in the diagnosis of PFO compared to autopsy, cardiac surgery, and/or catheterisation yielded a weighted sensitivity of only $89 \% .{ }^{45}$ Inability to perform an adequate Valsalva manoeuvre during transoesophageal echocardiography is probably responsible ${ }^{46,47}$ (Supplementary Figure 1). Nonetheless, c-TOE is necessary to characterise the PFO and stratify the risk in the diagnostic phase, ${ }^{31,48-52}$ and systematic reporting of a set of parameters could help in guiding assessment (Table 2). Bleeding, aspiration, or oesophageal perforation are rare TOE complications. ${ }^{53}$

In our updated meta-analysis of 29 studies comparing contrastenhanced transcranial Doppler (c-TCD) with c-TOE across 2, 751 patients (Supplementary Appendix 3, Supplementary Appendix 4, Supplementary Figure 19), c-TCD had a sensitivity of $94 \%$ and a specificity of $92 \%$ (Supplementary Figure $2 A$ ) with an area under the receiver operating curve (AUC) of 0.97 (Supplementary Figure 2B). This meta-analysis was limited by the low quality of evidence (Supplementary Table 2) and by the inconsistency across studies, being $67 \%$ for sensitivity and $73 \%$ for specificity. In a previous meta-analysis, the specificity of c-TCD was increased to $100 \%$ when the threshold for a positive shunt was increased to 10 high-intensity transient signals. ${ }^{54}$

We also performed an original meta-analysis of 13 studies across 1 , 360 patients comparing contrast-enhanced transthoracic echocardiography (c-TTE) against c-TOE (Supplementary Appendix 3, Supplementary Appendix 4, Supplementary Figure 20). c-TTE was only $88 \%$ sensitive and 82\% specific with an AUC of 0.91 (Supplementary Figure 3A), a severe inconsistency among studies (Supplementary Figure 3B) and a low quality of evidence (Supplementary Table 2). A recent meta-analysis also showed superior overall diagnostic yield of c-TCD compared to c-TTE. ${ }^{55}$

At present, grounded on the accrued low-quality evidence, no technique can be considered a gold standard and, in most cases, a precise diagnosis of PFO needs the combined use of different techniques, prescribed according to their different characteristics. As first-line investigations must warrant accuracy by minimising false negative screenings, we propose a diagnostic algorithm in Figure 1 that can be adapted to satisfy disparate clinical and logistic needs.

\section{Assessment of the role of a PFO in left circulation embolism}

A PFO is seen in $\sim 25 \%$ of the general population and may therefore coexist by chance in a patient with an unexplained left circulation embolism. Due to the complexity and number of the variables influencing the process, and the low scientific quality of the related literature, no position can be expressed regarding the assessment of the role of a PFO in a 
Table I Summary of statements

\begin{tabular}{|c|c|c|c|}
\hline Position statements & $\begin{array}{l}\text { Strength of } \\
\text { the statement }\end{array}$ & $\begin{array}{l}\text { Level of } \\
\text { evidence }\end{array}$ & Ref. \\
\hline \multicolumn{4}{|l|}{ General management of PFO-associated syndromes } \\
\hline Interdisciplinary assessment and decision making should be done & Strong & C & - \\
\hline $\begin{array}{l}\text { The decision making should be done taking into account an estimation of } \\
\text { the individual: } \\
\text { a. Probability of a causal role of the PFO in the clinical picture } \\
\text { b. Risk of recurrence }\end{array}$ & Strong & $\mathrm{C}$ & - \\
\hline $\begin{array}{l}\text { Individual risk stratification should take into account clinical, anatomical } \\
\text { and imaging characteristics }\end{array}$ & Strong & $\mathrm{C}$ & - \\
\hline $\begin{array}{l}\text { Shared decision making should be documented in an open, individualised, } \\
\text { informed consent }\end{array}$ & Strong & C & - \\
\hline $\begin{array}{l}\text { Decision aids and narrative tools are suggested to enhance patients' } \\
\text { involvement }\end{array}$ & Conditional & C & $38-43$ \\
\hline $\begin{array}{l}\text { Standardised definitions of candidate events should be adopted in } \\
\text { research and clinical settings }\end{array}$ & Strong & C & - \\
\hline \multicolumn{4}{|l|}{ PFO diagnosis } \\
\hline $\begin{array}{l}\text { To achieve the maximal accuracy in PFO diagnosis, the combined use of } \\
\text { different techniques is warranted }\end{array}$ & Strong & A & $\begin{array}{l}\text { 45, } 54,55+\text { Original meta-analyses } \\
\text { page } 4 \text { and Supplementary Appendix } 4\end{array}$ \\
\hline $\begin{array}{l}\text { The technique achieving the highest sensitivity should be used as a first- } \\
\text { line investigation in PFO diagnosis }\end{array}$ & Strong & C & - \\
\hline $\begin{array}{l}\text { c-TCD has a higher sensitivity than c-TTE as a first-line investigation to } \\
\text { detect a R-T-L shunt }\end{array}$ & Conditional & A & $\begin{array}{l}55+\text { Original meta- analyses page } 4 \\
\text { and Supplementary Appendix } 4\end{array}$ \\
\hline c-TTE has a lower sensitivity for small shunts than other techniques & Conditional & A & $\begin{array}{l}\text { Original meta-analyses page } 4 \text { and } \\
\text { Supplementary Appendix } 4\end{array}$ \\
\hline $\begin{array}{l}\text { c-TOE should be performed by experienced operators in PFO } \\
\text { assessment }\end{array}$ & Strong & C & $45-47$ \\
\hline A strict methodology should be used performing c-TOE & Strong & C & $46-47$ \\
\hline c-TOE should be performed to stratify the risk & Strong & C & $31,48-52$ \\
\hline
\end{tabular}

Table 2 PFO variables to be assessed for decision making and interventional treatment.

- PFO morphology: size, location, length of the tunnel

- Spatial relationship and distances between the PFO and the aortic root, vena cava, valves and the free walls of the atrium

- Comprehensive evaluation of the atrial septum, including inspection for atrial septal aneurysms, movement, and other atrial septal defects

- Presence/absence of a Eustachian valve and/or Chiari network

- Thickness of the septum primum and secundum

- Colour Doppler evaluation of the shunt at rest and after a Valsalva manoeuvre

quantitative way; therefore, this role should be evaluated with critical clinical judgement in an interdisciplinary collaboration between physicians, weighting the following different features on an individual basis. For a more detailed discussion of each of the following paragraphs please refer to Supplementary Appendix 4. Position statements are summarised in Table 3.

\section{Is it possible to estimate the likelihood of a PFO-mediated stroke?}

\section{Patient characteristics}

A meta-analysis of observational studies showed a stronger relative association of PFO with cryptogenic stroke in patients $<55$ years as compared to older patients. ${ }^{56}$ However, the association was also observed in older patients. ${ }^{13,57,58}$ The presence of other comorbidities or clinical risk factors for stroke does not, per se, exclude a pathophysiological role of PFO in cryptogenic embolism, though their absence increases the likelihood of its pathogenic role. ${ }^{59}$

\section{Imaging stroke pattern}

Neither the localisation nor type of infarct pattern in grey or white matter was specific for PFO embolism in observational studies. ${ }^{59-69}$ Cortical infarcts are usually considered embolic but a recent patient-level metaanalysis of RCTs plausibly suggests that non-cortical infarcts can also have an embolic origin. ${ }^{70}$

\section{Characteristics of the PFO}

An atrial septal aneurysm (ASA) and/or a moderate-to-severe shunt were strongly associated with a causal role of PFO in patients with cryptogenic stroke in observational and randomised studies. ${ }^{27-29,71-74}$ Other characteristics associated in randomised studies with a causal PFO are 


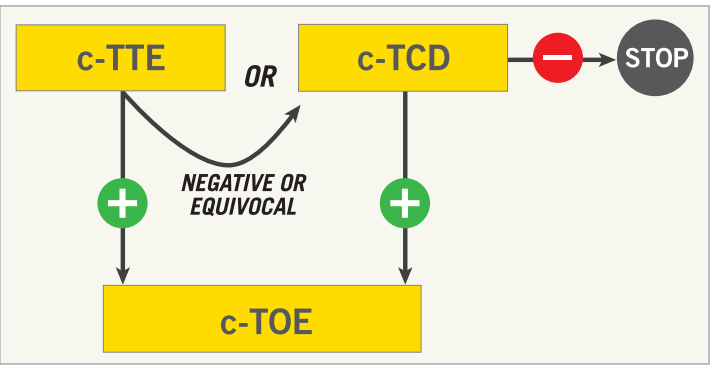

Figure I Algorithm for the diagnosis of PFO. c-TCD: contrastenhanced transcranial Doppler; c-TOE: contrast-enhanced transoesophageal echocardiography; c-TTE: contrast-enhanced transthoracic echocardiography; -negative test for the presence of right-to-left shunt; + positive test for the presence of right-to-left shunt.

large PFO size and atrial septal hypermobility. ${ }^{29}$ A Eustachian valve, Chiari network or a long PFO tunnel was suggested to be linked to PFOassociated strokes but only in retrospective studies. ${ }^{75,76}$ Other studies have failed to detect one or more of these associations, however, underlining the heterogeneity of phenotypes and the need to identify them.

\section{Clinical clues}

Candidate clinical clues have been addressed in retrospective studies and infrequently in prospective observational studies. Logically, conditions that strongly suggest paradoxical embolism in the presence of a PFO include the simultaneous or previous occurrence of pulmonary emboli ${ }^{18,80,81}$ or the documentation of a venous source of embolism around the time of stroke. Absence of evidence of venous thrombus is unhelpful because of frequent false negatives ${ }^{15,80,82,83}$ but immobilisation, recent major surgery, or an extended car or airplane journey implies possible venous clot development. Activity at the time of the stroke is also relevant - straining manoeuvres, obstructive sleep apnoea with strokeon-waking should be enquired for. ${ }^{81,84,85}$ Retrospective studies that have attempted to identify an association between inherited thrombophilia and PFO-related stroke have yielded conflicting results. ${ }^{86-89}$

The risk of paradoxical embolism (RoPE) score represents an attempt to assign a causal relationship probability to individual PFOs in the setting of stroke of unknown cause ${ }^{59}$ and may be useful in helping to guide management decisions. However, it should always be used in conjunction with other parameters because the quality of evidence of internal validation studies has been rated moderate at best (Supplementary Figure 15, Supplementary Table 3), and no large external validation studies have been published.

In addition, the RoPE score does not account for high-risk PFO features (e.g., septal aneurysm) that have been shown to correlate with higher risk of paradoxical embolisation.

\section{What is the risk of recurrence in a PFO- associated stroke?}

Meta-analyses of observational and/or randomised studies suggest that the annual recurrence rate on medical therapy ranges from $0 \%$ to $5.8 \%$ for stroke and from $0 \%$ to $14 \%$ for either stroke or transient ischaemic attack (TIA). ${ }^{90-92}$ This wide variability stresses the heterogeneity of phenotypes in these syndromes. Causes of recurrence can of course include non-PFO mediated mechanisms. ${ }^{93,94}$

Some predictors of stroke recurrence have been identified prospectively and retrospectively ${ }^{72,95-97}$ (Supplementary Figure 6). Supplementary Table 4 lists features that were statistically significant predictors in at least two studies. Atrial septal aneurysm anatomy is particularly predictive (Supplementary Appendix 4). In one study, ${ }^{98}$ a high Ddimer level on admission was an independent predictor of recurrent ischaemic stroke in patients with PFO. Therefore, at present, the individual evaluation of the risk of recurrence also cannot be quantitatively scored and should be based on interdisciplinary qualitative clinical evaluation.

\section{Unified diagnostic workup in left circulation thromboembolism}

A diagnostic workup should follow logical steps (Figure 2). Table 4 summarises position statements. Further details are provided in Supplementary Appendix 4.

The diagnostic process should always include interdisciplinary clinical assessments and appropriate imaging.

Identifying atrial fibrillation (AF) is important because recurrences of left circulation embolism are, in the majority of cases, due to left atrial appendage thrombosis instead of paradoxical embolism. However, AF can be difficult to detect. A routine 12-lead electrocardiogram (ECG) and either inpatient cardiac telemetry or 24-hour Holter monitoring are sufficient to diagnose permanent $A F$ and sufficiently long transient $A F$ episodes. However, randomised and observational studies showed that insertable cardiac monitors (ICM) are associated with an increased yield of paroxysmal AF diagnoses relative to standard monitoring also in cryptogenic stroke ${ }^{99-104}$ (Supplementary Appendix 4, Supplementary Figure 16). Therefore, in high-risk patients for AF, an ICM period of six months can be reasonably considered to rule out AF before deciding on PFO closure. ${ }^{105}$ In Figure 3 we propose a strategy based on risk stratification of patients to be applied with a critical clinical judgement (Supplementary Appendix 4). During ICM monitoring, patients should be maintained on medical therapy (see below). After six months, whatever the chosen treatment, the monitoring can be extended to the full duration of the ICM life to identify episodes of paroxysmal $A F,{ }^{106-112}$ to monitor the atrial thrombosis burden in arrhythmic patients, and to aid diagnosis in case recurrent ischaemia occurs.

\section{Medical and interventional management}

Further insights on each of the following paragraphs can be found in Supplementary Appendix 4.

PICO questions for the choice of therapy are summarised in Table 5 and Supplementary Appendix 5. Figure 4 summarises the flow of the choice of the therapy.

\section{Efficacy and safety of medical therapy}

A variety of medical treatments has been used, based upon data from secondary prevention studies for stroke in general and from studies on cryptogenic stroke in particular. No adequately dimensioned RCT has yet been published that has assessed the effectiveness of individual drugs specifically in PFO-associated cerebrovascular accidents.

Trials were almost exclusively observational with only one adequately dimensioned RCT comparing oral anticoagulants (OAC) and antiplatelet agents. One meta-analysis of RCTs showed a recurrent stroke rate of 1.27 events per 100 patient-years with drugs only. ${ }^{74}$ In our meta-analysis of the RCTs, the incidence of recurrent stroke on medical therapy was $4.6 \%$ after 3.8 years of follow-up (Supplementary Figure 4A, 
Table 3 Summary of statements on the assessment of PFO role in left circulation thromboembolism.

\begin{tabular}{|c|c|c|c|}
\hline Position statements & $\begin{array}{l}\text { Strength of } \\
\text { the statement }\end{array}$ & $\begin{array}{l}\text { Level of } \\
\text { evidence }\end{array}$ & Ref. \\
\hline PFO can play a pathogenic role in cryptogenic left circulation thromboembolism & Strong & A & $\begin{array}{l}9-29,51,112,132, \\
\text { Table } 5 \text { and } \\
\text { Supplementary Table } 7\end{array}$ \\
\hline $\begin{array}{l}\text { It is essential to evaluate the role of the PFO in any given left circulation } \\
\text { thromboembolism }\end{array}$ & Strong & A & Table 5 \\
\hline $\begin{array}{l}\text { No statement is possible regarding the quantification of the role of PFO in left cir- } \\
\text { culation thromboembolism }\end{array}$ & Strong & C & $13,18,27-29,57-98$ \\
\hline $\begin{array}{l}\text { The evaluation of the role of the PFO in left circulation thromboembolism should } \\
\text { be individualised with critical clinical judgement in an interdisciplinary collabora- } \\
\text { tion between physicians, weighting clinical, anatomical and imaging characteristics }\end{array}$ & Strong & $\mathrm{C}$ & $13,18,27-29,57-98$ \\
\hline \multicolumn{4}{|l|}{ Estimating the probability of a PFO being embolism-related } \\
\hline $\begin{array}{l}\text { No single clinical, anatomical or imaging characteristics are sufficient to make a } \\
\text { quantitative estimation of the probability of a PFO causal role }\end{array}$ & Strong & A & $\begin{array}{l}26-28,51,112,128,132 \\
\text { Table 5, 13, 59, 61, } \\
77-79,171\end{array}$ \\
\hline $\begin{array}{l}\text { When a PFO is considered to play a pathogenic role in an embolism, the episode } \\
\text { should not be classified as cryptogenic anymore }\end{array}$ & Strong & A & $\begin{array}{l}26-28,51,112,128,132, \\
\text { Table } 5\end{array}$ \\
\hline $\begin{array}{l}\text { The presence of other risk factors does not exclude a causative role of PFO; } \\
\text { however, it is more likely when patients are young and lack other risk factors }\end{array}$ & Strong & B & $13,56-59,78,79,90$ \\
\hline $\begin{array}{l}\text { Cortical infarcts are commonly embolic but, less frequently, also white matter } \\
\text { infarcts can be embolic }\end{array}$ & Strong & B & $59,60-63,70$ \\
\hline $\begin{array}{l}\text { No specific imaging pattern has been associated with a causal role of PFO in } \\
\text { stroke patients }\end{array}$ & Strong & C & $59-69,77$ \\
\hline $\begin{array}{l}\text { ASA, shunt severity and an atrial septal hypermobility can be linked to a causal } \\
\text { role of PFO }\end{array}$ & Strong & A & $\begin{array}{l}27-29,51,112,132, \\
\text { Table 5, Supplementary } \\
\text { Figure 5; 78, 79, 90, 122, } \\
170,171,71-74,91\end{array}$ \\
\hline $\begin{array}{l}\text { PFO sizes, presence of Chiari network or Eustachian valve can be linked to a } \\
\text { causal role of PFO }\end{array}$ & Conditional & C & $64,75,76,208,256$ \\
\hline $\begin{array}{l}\text { Deep vein thrombosis, immobilisation, long journeys, straining pre-stroke or } \\
\text { obstructive sleep apnoea can be linked to a causal role of PFO }\end{array}$ & Conditional & C & $81,84,85$ \\
\hline $\begin{array}{l}\text { Simultaneous pulmonary embolism and/or deep vein thrombosis strongly suggest } \\
\text { a causal role of PFO }\end{array}$ & Strong & $\mathrm{C}$ & $15,18,80-83$ \\
\hline $\begin{array}{l}\text { The role of thrombophilia cannot be generalised } \\
\text { The RoPE score should only be part of a comprehensive individual evaluation. }\end{array}$ & Strong & C & $86-89$ \\
\hline Further validation studies on the RoPE score are needed & Strong & B & $\begin{array}{l}59, \text { Supplementary } \\
\text { Table } 3\end{array}$ \\
\hline \multicolumn{4}{|l|}{ Estimating the risk of recurrences } \\
\hline The risk of recurrent embolism in unselected patients with PFO is low & Strong & A & $90-92,259$ \\
\hline No single variable allows a quantitative prediction of recurrences & Strong & A & $\begin{array}{l}94,95,26-28,51,112 \\
\text { 128, } 132, \text { Table } 5 \\
\text { Supplementary Table } 7\end{array}$ \\
\hline $\begin{array}{l}\text { Variables linked to a higher recurrence rate in PFO patients are: } \\
\text { - Atrial septal aneurysm and/or PFO diameter } \\
\text { - Older age } \\
\text { - Coagulation disorders } \\
\text { - Stroke at index } \\
\text { - D-dimer }>1,000 \text { at admission } \\
\text { - Acetylsalicylic acid use vs. OAC }\end{array}$ & Conditional & B & $72,95-98$ \\
\hline
\end{tabular}




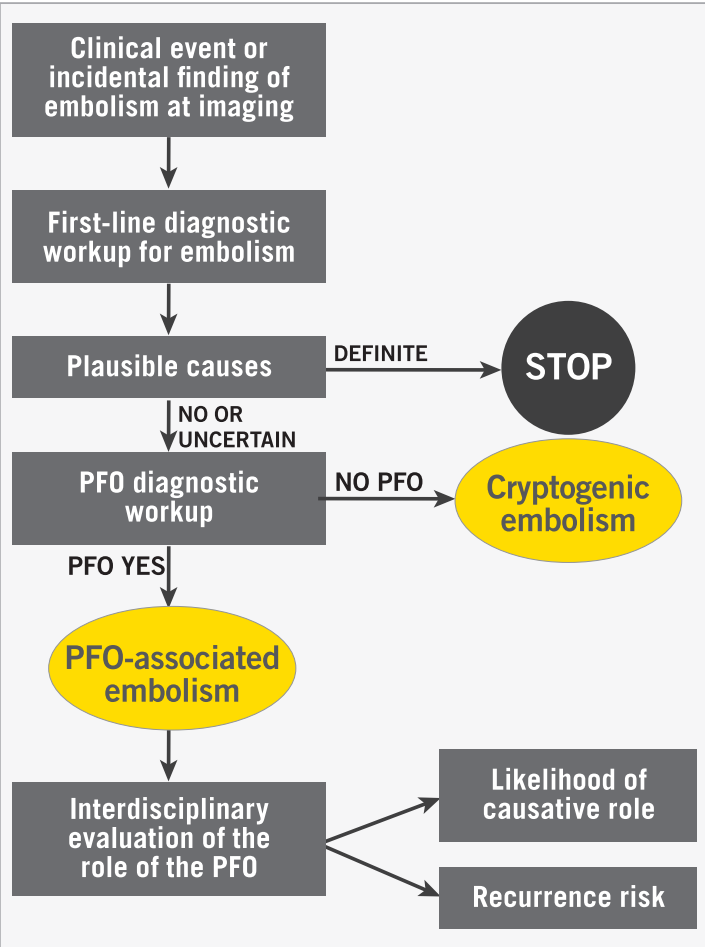

Figure 2 Algorithm for the diagnostic workup of cryptogenic left circulation thromboembolism.

Supplementary Figure 4B, Supplementary Appendix 3), whereas in a meta-analysis of observational trials the recurrence rate was $5 \%$ per year. $^{113}$

Despite a severe heterogeneity of results, the most recent metaanalysis including the randomised study is consistent with previous metaanalyses of observational studies, ${ }^{113-116}$ suggesting superiority of OAC over antiplatelet agents in the prevention of stroke (Supplementary Figure 7, Supplementary Appendix 3). Although the overall quality of the evidence in this meta-analysis was estimated to be very low (Supplementary Table 11), the superiority of OAC vs. antiplatelet agents was also evident when considering studies with multivariate adjustment only (Supplementary Figure 7). No data are available on persisting disability and quality of life.

Reports on safety have often been incomplete or have yielded inconsistent results. In a meta-analysis of observational studies, $1.1 \%$ of patients receiving medical therapy experienced a bleeding complication. ${ }^{113}$ This surprisingly low proportion of bleeding episodes can be explained by the young age of the patients and the short follow-up and, thus, must be interpreted with caution because most of these patients will undergo a lifelong medical therapy with an incremental risk of bleeding with age. Indeed, in our meta-analyses on PFO patients, an odds ratio (OR) of 4.57 was found for major bleeding with OAC relative to antiplatelet drugs (Supplementary Figure 8). A previous meta-analysis considering secondary prevention of stroke in general revealed that the potential benefit of OAC might be outweighed by the risk of both intracranial haemorrhage (OR 2.54) and major extracranial haemorrhage (OR 3.43). ${ }^{117}$ In this respect, direct oral anticoagulants (DOAC) may alter the risk-benefit ratio, ${ }^{118,119}$ although no data exist in these patients.

\section{Safety and efficacy profile of PFO closure Percutaneous procedure}

Primary technical success approaches $100 \%{ }^{78,113}$ and complete closure is seen in $93-96 \%$ at one year. ${ }^{122}$ The use of larger devices has a higher risk of residual shunts; ${ }^{113,123-125}$ the AMPLATZER ${ }^{\text {TM }}$ PFO Occluder (St. Jude Medical, St. Paul, MN, USA) may have lower residual shunt rates than other devices. ${ }^{123,125-130}$ Individual randomised data show a relative risk reduction of up to $80 \%$ for recurrent strokes. ${ }^{131,132}$ One meta-analysis of RCTs has shown the stroke recurrence rate to be 0.29 per 100 personyears $^{74}$ (Supplementary Appendix 3). In our study-level meta-analysis of RCTs with an average 3.8 years of follow-up, the incidence of recurrent stroke was $2 \%$ in the closure arms, and the number needed to treat (NNT) with PFO closure to prevent one stroke overall was 37 (95\% confidence interval [Cl]: 26 to 68) (Supplementary Figure 4A), and 21 in patients with high-risk PFO features ( $95 \% \mathrm{Cl}: 16$ to 61) (Supplementary Figure 5). Results on TIA and on death were neutral (Supplementary Figure 4C, Supplementary Figure 4D, respectively). An increase of the treatment effects over time can be expected. ${ }^{28,133,134}$ No data are available on persisting disability and quality of life.

Complications are summarised in Supplementary Table 5. Procedural complications had a $2.6 \%$ incidence in RCTs. ${ }^{74}$ The most frequent late complication is device thrombosis, which is seen in $1.0-2.0 \% .{ }^{135}$ Device embolism is a serious event and occurs at a rate of $0.9-1.3 \% .{ }^{135,136}$ Atrial wall erosions are serious events that have been reported anecdotally. The risk of long-term mortality or the need for cardiac surgery is less than one in 1,000. Minor complications occur only in 1.0-1.7\%.

The most frequent undesirable event following transcatheter percutaneous closure is AF in RCTs and observational trials. ${ }^{28,78,106-111,113}$ In a meta-analysis of RCTs, a $4.6 \%$ incidence was reported after 3.8 years of follow-up. ${ }^{74}$ In our meta-analysis, for incident $A F$, the overall number needed to harm $(\mathrm{NNH})$ was 25 (Supplementary Figure 9A), whereas beyond 45 days there was no increased risk for AF with PFO closure (Supplementary Figure 9B, Supplementary Figure 9C). The incidence of these events was lowest with the AMPLATZER PFO Occluder (Supplementary Figure 10). Interestingly, a statistically significant reduction of AF prevalence after percutaneous closure of PFO was also shown in other studies, suggesting some antiarrhythmic effect of the procedure. ${ }^{137}$

\section{Management after percutaneous closure}

No data on best management strategies after PFO closure are available. Position statements are summarised in Table 7.

\section{Drug treatments}

To decide on post-procedural therapy one should consider that: a) endocardialisation of the device can continue up to five years post implantation; ${ }^{128,138-140}$ b) one of the most frequent complications after closure is device thrombosis; and c) premature discontinuation of therapy may cause minor cerebrovascular events after PFO closure, as suggested by a marked trend towards association between duration of dual antiplatelet therapy after PFO closure and the incidence of TIA in our study-level meta-regression analysis (Supplementary Figure 11).

It is reasonable to decide on the post-procedural therapy according to the strategies used in RCTs. Overall, 5/6 RCTs prescribed or recommended a dual antiplatelet therapy in the first one to six months after closure, continuing with a single drug beyond two years in 3/4 RCTs that had a longer follow-up after that limit. In all positive trials, an antiplatelet therapy was prolonged for the entire duration of the study in the majority of 
Table 4 Summary of statements on the evaluation and treatment of concurrent diseases.

\begin{tabular}{|c|c|c|c|}
\hline Position statements & $\begin{array}{l}\text { Strength of } \\
\text { the statement }\end{array}$ & $\begin{array}{l}\text { Level of } \\
\text { evidence }\end{array}$ & Ref. \\
\hline \multicolumn{4}{|l|}{ AF rule-out strategy } \\
\hline $\begin{array}{l}\text { All patients should undergo a routine } 12 \text {-lead ECG and either in-patient cardiac telemetry } \\
\text { or } 24 \text {-hour Holter monitoring }\end{array}$ & Strong & B & 260,344 \\
\hline $\begin{array}{l}\text { In patients }>65 \text { years old with negative routine monitoring, it is reasonable to consider } \\
\text { ICM before deciding on PFO closure or permanent OAC }\end{array}$ & Conditional & C & $\begin{array}{l}99-102,105,166 \\
173,260-263\end{array}$ \\
\hline $\begin{array}{l}\text { ICM evaluation period in cryptogenic left circulation embolism should be at least } 6 \\
\text { months before deciding on PFO closure or permanent OAC }\end{array}$ & Conditional & B & $99-102,260-263,105$ \\
\hline $\begin{array}{l}\text { In patients } 55 \text { to } 64 \text { years old at risk for AF with negative routine monitoring, it is reason- } \\
\text { able to consider ICM before deciding on PFO closure or permanent OAC }\end{array}$ & Conditional & $\mathrm{C}$ & 173,264 \\
\hline $\begin{array}{l}\text { In patients }<55 \text { years old with } \geq 2 \text { high-risk factors for AF with negative routine monitor- } \\
\text { ing, it is reasonable to consider ICM before deciding on PFO closure or permanent OAC }\end{array}$ & Conditional & C & - \\
\hline Patients undergoing diagnostic procedures should be maintained on medical therapy & Strong & B & Table 6 \\
\hline Medical therapy should be decided according to the statements of this position paper & Strong & C & Table 6 \\
\hline $\begin{array}{l}\text { In patients with clear evidence of a causal PFO (e.g., simultaneous pulmonary embolism), } \\
\text { ICM can be withheld so as not to delay percutaneous closure }\end{array}$ & Strong & C & Table 5 \\
\hline $\begin{array}{l}\text { In patients undergoing ICM, the monitoring should be extended for the full duration of } \\
\text { the device life, regardless of the choice of therapy after } 6 \text { months }\end{array}$ & Strong & $\mathrm{C}$ & 102 \\
\hline \multicolumn{4}{|l|}{ Management of PFO in the presence of concomitant diseases } \\
\hline $\begin{array}{l}\text { Patients on temporary } \mathrm{OAC} \text {, on } \mathrm{OAC} \text { for pulmonary embolism or those considered at } \\
\text { high risk of recurrences despite } \mathrm{OAC} \text { may undergo } \mathrm{PFO} \text { assessment for possible closure }\end{array}$ & Conditional & C & 159,160 \\
\hline $\begin{array}{l}\text { Paroxysmal AF episodes }>30 \text { seconds detected with intermittent recordings, or } \geq 5 \\
\text { minutes during ICM can be considered sufficient to evaluate the patient for OAC accord- } \\
\text { ing to current guidelines on AF }\end{array}$ & Conditional & B & $163-168$ \\
\hline $\begin{array}{l}\text { ICM results should always be interpreted with other clinical characteristics in order to } \\
\text { weigh the AF embolic risk against the PFO embolic risk }\end{array}$ & Strong & C & 102 \\
\hline $\begin{array}{l}\text { Routine laboratory tests for prothrombotic states (thrombophilia testing) are not war- } \\
\text { ranted to indicate permanent OAC }\end{array}$ & Strong & C & 161,162 \\
\hline
\end{tabular}

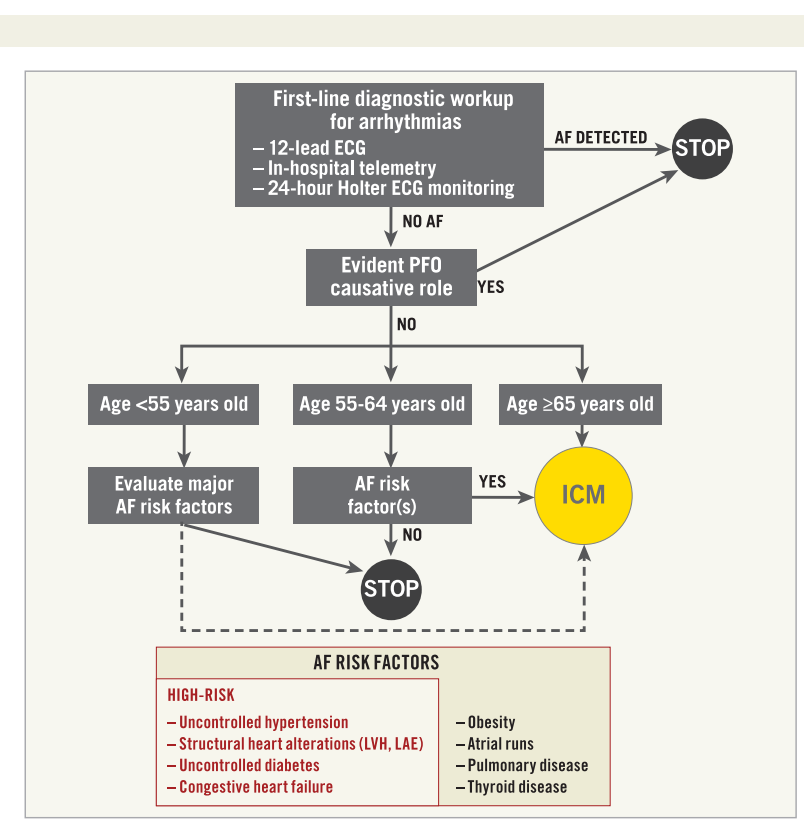

Figure 3 Flow chart for the screening of overt atrial fibrillation in cryptogenic left circulation thromboembolism. The cut-off ages of 55 and 65 years old have been chosen according to data from large epidemiological studies. ${ }^{166,173}$ Patients $<55$ years may be considered for ICM when they have high clinical suspicion of AF (i.e., $\geq 2$ high-risk factors for AF). ECG: electrocardiography; LAE: left atrium enlargement; LVH: left ventricle hypertrophy. 
Table 5 PICO question. Should percutaneous closure of PFO vs. medical therapy be used for secondary prevention of stroke or other left circulation thromboembolism in patients with high-risk PFO features?

\begin{tabular}{ll}
\hline Population & Secondary prevention of stroke, TIA, or other left circulation thromboembolism \\
Intervention & Percutaneous closure of PFO \\
Comparison & Medical therapy \\
Main outcomes & Stroke, TIA, death, bleedings, atrial arrhythmias
\end{tabular}

TYPE OF STATEMENT POSITION STATEMENTS
Strong statement for the intervention

The position of our societies is to perform percutaneous closure of a PFO in carefully selected patients aged from 18 to 65 years with a confirmed cryptogenic stroke, TIA, or systemic embolism and an estimated high probability of a causal role of the PFO as assessed by clinical, anatomical and imaging features.

The interventional procedure must be proposed to each patient evaluating the individual probability of benefit based on an assessment of both the role of the PFO in the thromboembolic event (Table 4) and the expected results and risks of a lifelong medical therapy. The role of the patient should be proactive, keeping in highest regard his/her values and preferences regarding outcomes and therapy trade-offs, and informing him/her about the uncertainties of their condition.

With the same shared decision-making approach, PFO closure can also be considered in patients $>65$ or $<18$ years of age, taking into account on a case-by-case basis the lack of evidence, the age-related confounders and additional risks of interventional and drug therapies.

Although no specific data are available to date, consistent with some guidelines on the topic, it seems justified to consider percutaneous closure in patients with a cryptogenic TIA, stroke, or systemic emboli that occurred while on therapy with OAC or antiplatelet agents.

The choice of device should take into consideration that most available evidence has been obtained with the AMPLATZERTM PFO Occluder and GORE ${ }^{\circledR}$ HELEX ${ }^{\circledR}$ Septal Occluder (not available anymore) or the GORE ${ }^{\circledR}$ CARDIOFORM Septal Occluder. The use of the latter should be balanced against a lower complete closure rate and a higher risk of AF as compared to medical therapy. The potential use of devices other than AMPLATZER and CARDIOFORM, and the inherent risks, should also be part of the shared decision making with patients, in the light of technical, anatomical, and clinical features.

\section{Overall justification}

The last, comprehensive, study-level meta-analyses incorporating the most recent randomised trials on patients aged 1865 years with prior cryptogenic stroke or TIA showed superiority of PFO closure over medical therapy for the prevention of stroke in the first 5 years after the procedure (Supplementary Figure 17, Supplementary Figure 4A). One exploratory analysis of one of these trials extended to a longer follow-up supports a growing benefit of percutaneous closure over medical therapy after that time limit.

The CLOSE, and the early-terminated DEFENCE-PFO trials performed in characterised patients with confirmed cryptogenic stroke and high-risk PFO features, and the REDUCE trial which also enrolled higher-risk patients as compared to previous trials, are the main drivers of this evidence (Supplementary Figure 4B and Supplementary Figure 5). The difference in results between studies enrolling higher-risk PFO patients and those enrolling unselected

patients with prior cryptogenic cerebral accidents stresses the existence of higher- and lower-risk phenotypes of patients that need to be characterised before deciding on the therapy. This finding is furthermore supported by the cost- effectiveness analysis which demonstrated a benefit over 15 years only in high-risk patients. However, the significant effect in some subgroups, the heterogeneity still present at subgroup analysis even in high-risk patients, and the individual study limitations (Supplementary Appendix 4) enforce the need for carefully informed choices which must be shared with patients and tailored to their personal values and preferences.

\section{Detailed justification}

Problem. PFO-related stroke is an important health problem; therefore, its secondary prevention is a priority. Unfortunately, its management is problematic because high-quality data are lacking in this very heterogeneous class of patients. Nonetheless, the possibility of an efficient secondary prevention should be granted without causing harm with unnecessary treatments. Given the very disparate practices that exist within the medical community in this regard, it is urgent that clinicians follow a balanced approach that is based upon the present level of knowledge, while waiting for more conclusive evidence on better classified populations of patients.

Desirable effects. Our study-level meta-analysis on the 6 RCTs showed a clear superiority of PFO closure over medical therapy in terms of reducing the incidence of stroke recurrence (Supplementary Figure 4A). The two previously published meta-analyses on the 6 RCTs, all of the first five RCTs (hence excluding the DEFENSE-PFO trial) and the

Continued 
Table 5 Continued

\begin{tabular}{ll}
\hline Population & Secondary prevention of stroke, TIA, or other left circulation thromboembolism \\
Intervention & Percutaneous closure of PFO \\
Comparison & Medical therapy \\
Main outcomes & Stroke, TIA, death, bleedings, atrial arrhythmias
\end{tabular}

highest-quality, patient-level meta-analysis of the first three published RCTs are consistent with our results. Two meta-analyses of comparative observational trials are in keeping with these results (Supplementary Table 7).

Undesirable effects. Interventional treatment does not imply higher complication rates, with the exception of a higher frequency of AF after percutaneous closure relative to medical therapy (Supplementary Figure 9). However, the higher risk of AF with closure versus medical therapy was considerably lowered (Supplementary Figure 10) if an AMPLATZER PFO Occluder was used. In the REDUCE trial using the GORE HELEX or CARDIOFORM septal occluders, the incidence of AF was $6.6 \%$ at 5 years, a large proportion of which were only intraprocedural or periprocedural arrhythmias. Bleeding complications were similar in the young cohorts of patients enrolled in RCTs in the short term; however, long-term follow-up data are missing in patients undergoing lifelong medical treatments, which are likely to increase the risk of haemorrhage as patients grow older.

Certainty of evidence. The consistent results of all the meta-analyses performed so far were confirmed when considering OR, RR and AR, even performing sensitivity analysis, and also when including CLOSURE I, the most outdated trial.

To date, despite several limitations of individual studies which implied an overall low score in the certainty of evidence (Supplementary Table 9, Supplementary Table 10), in patients with high-risk PFO features the certainty is higher, as shown by the reduction in heterogeneity in meta-analyses and by the recently published sequential analysis of the risk of recurrent stroke74. Therefore, future studies are not likely to impact on the certainty of evidence, at least in highrisk populations.

Values. Large variations in preferences of patients indicate the need for tailored informed consent and the explicit evaluation of therapeutic trade-offs with individual patients.

Balance of effects. The NNT with percutaneous closure obtained in RCTs outweighed the NNH for atrial fibrillation after percutaneous closure, especially when an AMPLATZER PFO Occluder was used and when patients with higherrisk PFO were considered. Moreover, based on United States estimates, the cost-effectiveness analysis favours over 15 years percutaneous closure in patients with high-risk PFO features and with the use of an AMPLATZER PFO Occluder.

SUBGROUP CONSIDERATIONS
In published randomised studies, the age of patients was $\leq 65$ years and 18 years. The DEFENSE-PFO trial, strongly positive for PFO closure over medical therapy in the prevention of recurrent stroke, did not have age limitations for enrolment and randomised patients aged up to 66 years old29.

In our study-level meta-analysis of the 6 RCTs, a statistically significant improvement in stroke recurrence with percutaneous closure was observed only versus antiplatelet therapy (Supplementary Figure 12A), whereas OAT yielded a similar risk of recurrence (Supplementary Figure 12B, Supplementary Figure 12C). Moreover, no differences were noted regarding the outcomes of different pooled clinical inclusion criteria regarding the index event (Supplementary Figure 13). However, some of the previous meta-analyses on the first 5 RCTs consistently found that patients with moderate-to-severe shunt size experienced enhanced outcomes with percutaneous closure relative to medical therapy78, $79,90,122,170,171$. Nonetheless, patients with ASA were associated with better outcomes with percutaneous closure than with medical therapy only in some171, 172 but not in other meta-analyses78, 79. In our meta-analysis, we found that patients with high-risk PFO features (ASA, hypermobility of atrial septum, moderate-to-severe shunt, or large PFO size) reported enhanced outcomes with percutaneous closure relative to medical therapy, whereas in patients with low-risk PFOs there was no additional benefit with PFO closure vs. medical therapy (Supplementary Figure 4B, Supplementary Figure 5).

In our most recent analysis, no device was associated with statistically significant enhanced efficacy versus medical therapy as compared to other devices (Supplementary Figure 14). The risk of new-onset atrial fibrillation was similar with the AMPLATZER PFO Occluder and medical therapy while it was higher for the GORE CARDIOFORM device when compared with medical therapy (Supplementary Figure 10).

In some meta-analyses other subgroups experienced enhanced outcomes with percutaneous closure relative to medical therapy. These subgroups include males70, 78, 79, 90 and age <45 years old78, 79, 90. However, these findings were not confirmed in another meta-analysis171. Finally a single previous meta-analysis supported patients with a history of migraines or non-cortical infarcts as having better outcomes with percutaneous closure as compared to medical therapy 70 . 
Table 5 Continued

\begin{tabular}{ll}
\hline Population & Secondary prevention of stroke, TIA, or other left circulation thromboembolism \\
Intervention & Percutaneous closure of PFO \\
Comparison & Medical therapy \\
Main outcomes & Stroke, TIA, death, bleedings, atrial arrhythmias
\end{tabular}

IMPLEMENTATION CONSIDERATIONS

\section{MONITORING AND EVALUATION}

RESEARCH

PRIORITIES
PFO closure incurs procedural cost. However, cost-effectiveness studies showed that PFO is associated with economic and QUALY gain after 15 years, provided that the procedure was performed in high-risk patients. Performing the procedure in unselected patients translates into a sharp decrease in cost-effectiveness.

Moreover, procedural costs and procedure times may be decreased with the use of sedation instead of general anaesthesia or of intracardiac echocardiography versus transoesophageal echocardiography, thereby eliminating the need for an anaesthesiologist.

Each neurological index event should be confirmed by a neurologist or a stroke physician. The cardiologist and the stroke physician must come to the conclusion that the stroke or TIA was cryptogenic and communicate in order to reach consensus regarding therapeutic decisions. Patients should be actively involved at all stages of management and their contribution to choices should be documented.

- To verify the existence of additional risk factors and their cut-offs for prediction of events in strict epidemiological series.

- To identify new high-risk phenotypes encompassing different clusters of clinical, anatomical and biological characteristics in prospective observational trials (systems and precision approaches) and to perform new randomised trials in these populations.

- To design adequately dimensioned RCTs comparing single medical therapies (vitamin K antagonists or DOAC) with percutaneous closure in patients with higher-risk PFO-related left circulation embolism.

- To assess outcomes of percutaneous closure vs. OAC.

- To assess long-term outcomes (>5 years) with different treatments.

- To address the evaluation of persisting disability and quality of life with different treatments.

- To design prospective registries to evaluate practices and outcomes in the real world.

- To obtain new, cost-effectiveness analyses based on contemporary practices.

- To obtain quantitative and qualitative data on patient preferences and values in the setting of cryptogenic stroke or systemic embolism with PFO.

- To obtain data on the effectiveness and efficacy of organisational models to manage patients with cryptogenic stroke/ systemic emboli. patients (in 2/4 studies it was prescribed for five years). In one negative trial, only $50 \%$ and $41 \%$ of patients were still taking an antiplatelet therapy after one and five years, respectively. ${ }^{132}$

\section{Delayed complications}

Supplementary Table 5 displays the main tools available to detect complications. At present, no relationship between PFO patency after closure and the incidence of recurrence has been found (Supplementary Table 6), ${ }^{124,141-147}$ but studies were small, often plagued by partially incomplete follow-up, and problematic regarding shunt detection accuracy. ${ }^{139}$ Also, a persistent shunt after closure may reveal other sources of paradoxical embolism which were missed during the diagnostic phase. ${ }^{148}$

No high-quality data are available to guide the optimal management of a residual moderate-to-severe PFO patency. The literature on acute and long-term results after repeat device implantation for a residual shunt is scarce, but retrospective evaluations are encouraging. ${ }^{149-156}$

Empirically, antibiotic prophylaxis against endocarditis before an invasive procedure or surgical intervention should also be considered routinely in all cases within the first six months after the implantation and, probably, beyond six months in patients with a residual shunt.

\section{Surgical closure of PFO}

There are no current indications for surgical closure of a PFO as first-line treatment. Closure of incidental PFOs is usually undertaken during valvular surgery or in the rare cases when surgery is indicated for other conditions in which the PFO plays a role, such as a straddling thrombus in the PFO, or seldom when complications of percutaneous closure occur which cannot be managed by percutaneous means.

\section{Management in the presence of concomitant diseases} Position statements are summarised in Table 4.

In the setting of hypercoagulability, deep vein thrombosis and/or pulmonary embolism, ${ }^{159}$ PFO closure may be considered when there is the need for only temporary OAC or a high risk of recurrence despite permanent OAC, particularly in pulmonary embolism, where PFO was reported to be an independent predictor of new brain lesions in the follow-up, despite optimal OAC. ${ }^{160}$

Routine laboratory tests for prothrombotic states (thrombophilia testing) are not generally warranted to guide the need for permanent OAC. ${ }^{161,162}$

Although no study has assessed this issue as yet, it is reasonable that excluding patients with AF from PFO closure and treating them with 
Table 6 PICO question. Should oral anticoagulants (OAC) vs. antiplatelet therapy be used for secondary prevention of stroke or other left circulation thromboembolism?

\begin{tabular}{ll}
\hline Population & Secondary prevention of stroke or other left circulation thromboembolism \\
Intervention & OAC \\
Comparison & Antiplatelet therapy \\
Main outcomes & Stroke; major bleedings
\end{tabular}

TYPE OF STATEMENT

POSITION STATEMENT

\section{JUSTIFICATION}

\section{SUBGROUP CONSIDERATIONS IMPLEMENTATION CONSIDERATIONS}

\author{
MONITORING AND \\ EVALUATION
}

RESEARCH PRIORITIES
Conditional statement for either the intervention or the comparison

In patients in whom a medical therapy only is chosen, the position of our scientific societies is to choose the specific drugs weighing the individual risk of bleeding against the risk of PFO-related stroke recurrence, in close connection with the patient. Long-term OAC with vitamin K antagonists may be preferred if: a) the patient has a low haemorrhagic risk, b) a probable good therapeutic compliance is foreseen, and c) a proper anticoagulant monitoring can be guaranteed. In patients in whom these conditions are not satisfied, or the risk of stroke recurrence is deemed low, an antiplatelet therapy should be prescribed. Reassessment of the risk/benefit ratio should be performed on a regular basis, especially with advancing age and the increase in comorbidities which can affect both risk and benefit issues. No position can be expressed for DOAC, although intuitively their reduced bleeding risk compared with vitamin $\mathrm{K}$ antagonists in other clinical conditions is appealing.

\section{Overall justification}

The randomised CLOSE trial shows a statistically non-significant reduction of stroke with OAC as compared to antiplatelet therapy. However, a single trial enrolling only 300 patients reporting outcomes with wide confidence intervals cannot be considered conclusive. Meta-analyses consistently indicate a statistically significant reduction in the risk of stroke with OAC as compared to antiplatelet therapy, at the cost of a significantly higher risk of major bleeding. However, the overall uncertainty of the evidence remains very high (Supplementary Table 11) and the inconsistency across studies is severe (Supplementary Figure 7).

Therefore, only a conditional statement for either OAC or antiplatelets can be expressed, with the choice between them being guided by individual safety and expected risk of recurrence variables.

\section{Detailed justification}

Desirable effects. The randomised CLOSE trial shows a statistically non-significant reduction of stroke with OAC as compared to antiplatelet therapy. Our meta-analysis indicates a statistically significant reduction of the odds ratio for stroke of approximately $12 \%$ with OAC over antiplatelet therapy (Supplementary Figure 18). These results are in keeping with previous meta-analyses.

Undesirable effects. An approximately 5-fold higher risk of major bleeding emerged from our meta-analysis with OAC as compared to antiplatelet therapy. Also, these results are in line with previous analysis.

Certainty of evidence. The certainty of evidence is very low, because the results are mainly derived from non-randomised comparisons (Supplementary Table 11), and the included randomised trial, enrolling only approximately 300 patients, reported wide confidence intervals in effect estimates. Therefore, further RCTs will probably impact on effect estimates.

Values. Patients undergoing secondary pharmacological prevention for stroke appear to accept higher risk of bleeding if a considerable certitude can be provided regarding the prevention of stroke.

Balance of effects. The balance of desirable and undesirable effects of therapy varies according to the expected benefits of the therapy, as the risk of bleeding appears to be homogenous across studies. Therefore, therapy should be as individualised as possible.

Feasibility. Feasibility of implementation of a safe OAC regimen with vitamin K antagonists is largely depend ent on availability of monitoring facilities of proper anticoagulation and on the possibility of accessing them by patients.

No subgroup consideration can be derived from the accrued data. However, given the inconsistency of the studies and the variability of results, subgroups should be identified for new study.

No cost-effectiveness studies have been performed in this field. However, as the costs of OAC and antiplatelet therapy are low, the cost-effectiveness profile is dependent mainly on the costs of adverse events in the follow-up. The available evidence shows that bleeding complications increase with age, rendering even more uncertain the cost-effectiveness of this therapy in the long term.

In antithrombotic therapy the risk/benefit ratio is highly dependent on time. It is therefore advised to reassess risks and benefits of the chosen therapy on a regular basis, especially with advancing age and the increase in comorbidities. Local registries for prospective evaluations of outcomes are strongly encouraged.

- To assess more precise risk factors and their cut-offs for prediction of events. 
Table 6 Continued

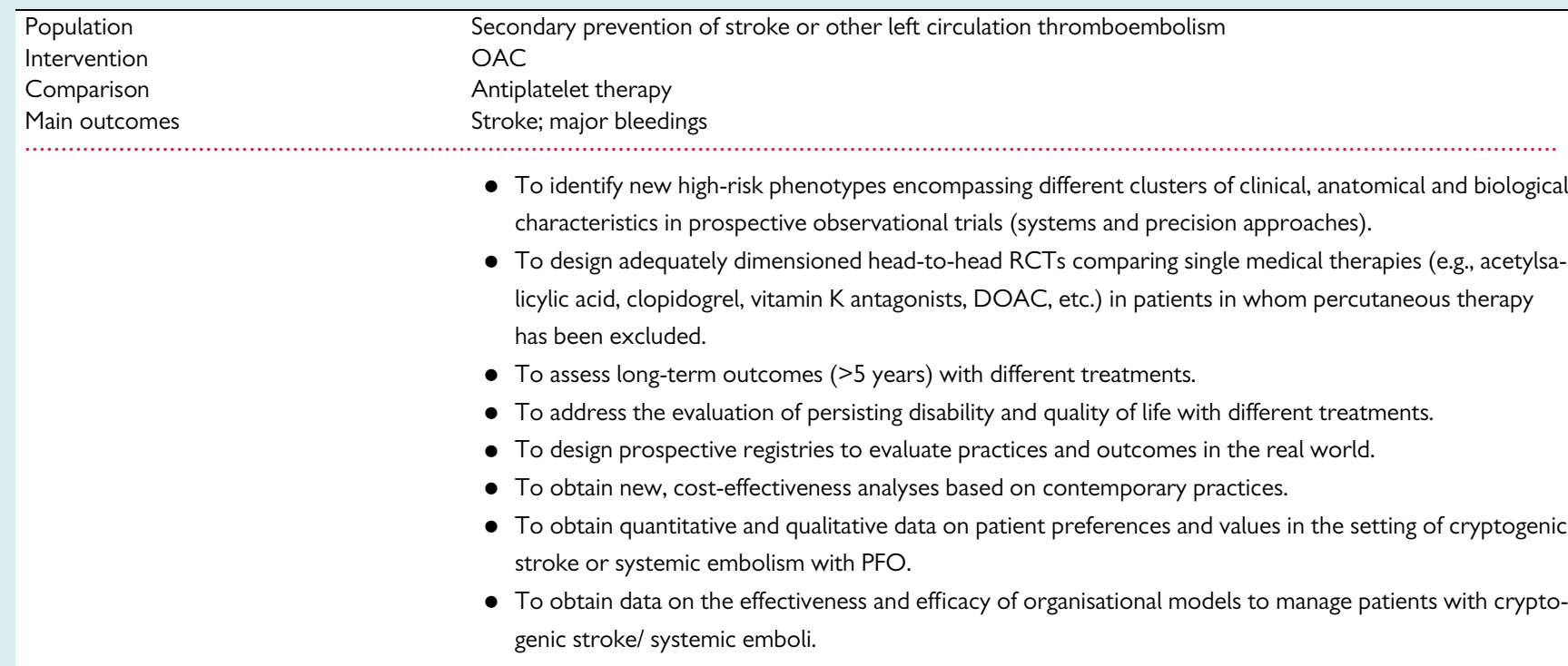

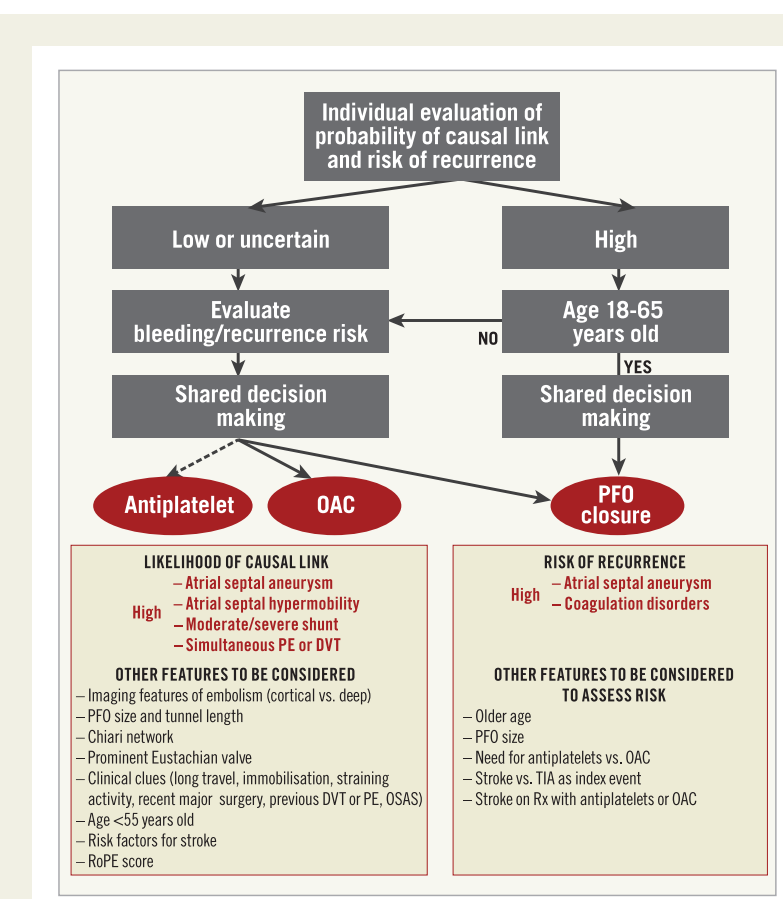

Figure 4 Treatment algorithm for secondary prevention of left circulation cryptogenic thromboembolism. DVT: deep vein thrombosis; OAC: oral anticoagulants; OSAS: obstructive sleep apnoea syndrome; PE: pulmonary embolism; Rx: therapy; TIA: transient ischaemic attack. permanent OAC should translate into an increased effectiveness of secondary prevention of left circulation thromboembolism. However, as in the CRYSTAL-AF study, a higher incidence of AF at ICM did not translate into a higher stroke incidence; ${ }^{102}$ the presence of short bursts of AF on an ICM may carry a lower pathogenic value than a high-risk PFO. Therefore, the burden of AF should be weighed against the burden of PFO by considering other clinical characteristics to decide for or against PFO closure. For patients with paroxysmal AF, there is uncertainty regarding the duration of arrhythmic episodes which increases the risk of embolism. According to the HRS/EHRA/ECAS expert consensus statement on $A F$ ablation, $A F$ episodes $\geq 30$ seconds constitute clinically significant $A F{ }^{163}$ During prolonged monitoring, episodes of $A F \geq 5$ minutes have a predictive value for embolism. ${ }^{164-168}$ These criteria should be combined with a thromboembolic score to evaluate the need for OAC. $^{169}$

\section{Conclusions}

The management of patients with cryptogenic left circulation thromboembolism and PFO has been controversial, giving rise to heterogeneous strategies across different local realms in Europe. Based on the best available evidence, we were able to reach, in this interdisciplinary position paper, a consensus among eight European scientific societies on key diagnostic, therapeutic and research issues, from the index event to follow-up. It was possible to express strict position statements based on randomised trials for some therapeutic aspects, whereas other aspects were often based on limited and nonrandomised data. This position paper provides the first largely shared approach for a rational PFO management based on the best available 
Table 7 Summary of statements on the management after percutaneous closure of PFO

\begin{tabular}{|c|c|c|c|}
\hline Position statements & $\begin{array}{l}\text { Strength of } \\
\text { the statement }\end{array}$ & $\begin{array}{l}\text { Level of } \\
\text { evidence }\end{array}$ & Ref. \\
\hline \multicolumn{4}{|l|}{ Drug therapy and follow up after percutaneous closure } \\
\hline It is reasonable to propose dual antiplatelet therapy for 1 to 6 months after PFO closure & Conditional & A & $\begin{array}{l}27,29,51,112,132 \\
\text { Supplementary Figure } 11\end{array}$ \\
\hline We suggest a single antiplatelet therapy be continued for at least 5 years & Conditional & $\mathrm{C}$ & $\begin{array}{l}27-29,51,112,132 \\
128,138-140\end{array}$ \\
\hline $\begin{array}{l}\text { The extension of the therapy with single antiplatelet beyond } 5 \text { years should be based on } \\
\text { the balance between patient's overall risk of stroke for other causes and haemorrhagic } \\
\text { risk }\end{array}$ & Strong & C & - \\
\hline The choice of the type of antiplatelet drug in the follow-up is currently empiric & Strong & A & $27-29,51,112,132$ \\
\hline $\begin{array}{l}\text { The value of residual shunt after percutaneous closure cannot be deduced from available } \\
\text { studies }\end{array}$ & Strong & C & $124,141-47$ \\
\hline Systematic, high-quality data on follow-up are needed & Strong & C & - \\
\hline $\begin{array}{l}\text { To obtain comparable data we propose to perform: } \\
\text { a. a TTE prior to hospital discharge } \\
\text { b. c-TCD at least once beyond six months to assess effective PFO closure and there- } \\
\text { after, if residual shunt persists, annually until closure } \\
\text { c. c-TOE or c-TTE in case of severe residual shunt at c-TCD, or recurrent events, or } \\
\text { symptoms during follow-up }\end{array}$ & Conditional & C & $\begin{array}{l}\text { 124, } 141-147, \\
55+\text { Original meta-analyses } \\
\text { page } 4 \text { and Supplementary } \\
\text { Appendix } 4\end{array}$ \\
\hline $\begin{array}{l}\text { Patients should undergo antibiotic prophylaxis for any invasive procedure performed in } \\
\text { the first six months from PFO closure }\end{array}$ & Conditional & $\mathrm{C}$ & - \\
\hline
\end{tabular}

evidence. This may help physicians to offer coherent strategies throughout Europe and focus the research on high-priority subjects.

\section{Supplementary material}

Supplementary material is available at European Heart Journal online.

\section{Acknowledgements}

We are indebted to Marielle De La Torre, Jenny Pena, Laurence Fani and the entire Specialty Centre Division of the European Association of Percutaneous Cardiovascular Interventions for their invaluable and highly professional support throughout the phases of development of this position paper.

Conflict of interest: $H$. Sievert reports institutional fees from Carag and Lifetech, outside the submitted work. B. Dalvi reports being a consultant to Abbott. B. Meier declares a conflict of interest in the form of speaker fees received from Abbott. M. Chessa reports personal fees from Abbott and Occlutech. D. Toni reports personal fees from Boehringer Ingelheim, Bayer, Pfizer Bristol-Myers Squibb, Daiichi Sankyo, and Medtronic. P. Scacciatella reports personal fees from Abbott Medical and Gore Medical. J. Thomson reports being a proctor and consultant for Abbott Medical and Gore Medical. D. Hildick-Smith reports having consultancy/advocacy for Abbott, Gore and Occlutech. D. Sibbing reports personal fees outside the submitted work from Roche Diagnostics, Daiichi Sankyo, Eli Lilly, Bayer Healthcare, Sanofi, Pfizer and AstraZeneca. S. Kasner reports personal fees from Bristol-Myers Squibb, Boehringer Ingelheim, Medtronic and AbbVie, grants from W.L. Gore, and grants and personal fees from Janssen and Bayer, outside the submitted work. G. Biondi-Zoccai reports having been a consultant for Abbott Vascular and Bayer. J. Carroll reports personal fees from AGA Medical, St. Jude Medical, and Abbott. The Chairman of the task force and all the other authors have no conflicts of interest to declare. The Guest Editor has no conflicts of interest to declare.

\section{References}

The references can be found in the Supplementary data document. 